Jpn. J. Genet. (1981) 56, pp. 19-26

\title{
Studies on the genetical and biochemical properties of the Kiuki mutant of the silkworm,
}

\author{
Bombyx mori1)
}

\author{
By Toshio Mazda, Motoo Tsusút, Sakae Sakate* \\ and Hiroshi DoIRA** \\ Biological Laboratory, Kitasato University, Sagamihara City 228, \\ * Sericultural Experiment Station, Yatabe, Tsukuba, Ibaraki 305 \\ and **Institute of Silkworm Genetics, Faculty of Agriculture, \\ Kyushu University, Fukuoka 812
}

(Received February 28, 1980)

\begin{abstract}
Genetic studies of the Bombyx mori Kiuki mutant were made on the segregation of the $\mathrm{F}_{2}$ and $\mathrm{BF}_{1}$ phenotypes. Assay of sepialumazine in these phenotypes revealed that the Kiuki mutant is an incomplete dominant. A symbol $\mathrm{Sel}$ is given to the mutant gene. Enzymatic studies on $\mathrm{F}_{2}$ progeny showed that the yellow larval integument colour characteristic of the mutant, which is due to accumulation of sepialumazine, is dependent on the activity of sepiapterin deaminase. Linkage test showed that the $\mathrm{Sel}$ is independent of the so far established 23 linkage groups but represents one of the still unknown group among remaining five chromosomes.
\end{abstract}

\section{INTRODUCTION}

Genetic studies of the Bombyx mori yellow mutant lemon have been conducted for many years (Ogura 1922). Tsujita and Sakaguchi (1955) showed an allelomorphism in the lemon lethal, lemon and normal strains by genetic analysis of the $F_{1}$ hybrid between the homozygous lemon (lem/lem) and the heterozygous lethal yellow $\left(+/ l e m^{l}\right)$. A dominant recessive relation $+^{l e m}>$ $l e m>$ lem $^{l}$ was also reported. Tsujita (1961) explained the properties of the multiple allele by stating that the lemon lethal mutant was completely deficient in sepiapterin reductase and the lemon mutant had a decreased level of the enzyme. Subsequent studies of the lemon mutant, however, have failed to detect any sepiapterin reductase activity (Matsubara et al. 1963; Mazda et al. 1980).

Tsusué and Akino (1965) isolated a major yellow pigment from the integument of lemon silkworms and identified it as 7,8-dihydro-2-amino-4-hydroxy6-lactylpteridine (sepiapterin). Goto et al. (1966) later showed that a minor yellow pigment in this same strain was 7,8-dihydro-6-lactyllumazine (sepialumazine). Tsusué (1967) reported the occurrence of an enzyme in lemon larvae

1) This paper is dedicated to Professor emeritus Daigoro MoRIwakI in recognition of his longlasting leadership in genetics, on the occasion of his seventyseventh birthday. 
which catalyzed the deamination of sepiapterin to form sepialumazine. Detailed properties of the enzyme were revealed by subsequent work of Tsusué (1971). The occurrence of this same enzyme as well as its product in the normal type silkworm larvae was shown by Tsusué and Mazda (1977).

Another yellow coloured silkworm mutant, Kiuki, has a rather pale yellow hue when compared with lemon mutant larvae. Although the mutant silkworm have been reared for many years, the properties of the mutant had remained unknown until recent work (Mazda et al. 1978) showed that the chemical structure of the yellow pigment is that of sepialumazine. A recent paper (Mazda et al. 1980) has showed that sepialumazine is the cause of the pale yellow colour in Kiuki larvae. In the present paper we will show that the mutant Kiuki is an incomplete dominant as based on assays of sepialumazine in the larval integument of hybrid larvae. Studies on the linkage relation of the gene in the mutant will also be described.

\section{MATERIALS AND METHODS}

Bombyx mori mutants and mating experiments. The Kiuki mutant has been reared in the Sericultural Experiment Station since 1945. Linkage studies were made using the autosomal marker gene described bellow: $Y(2), Z e(3)$, $L(4), o c(5), E^{E t}(6), q(7), s t(8), I-a(9), w-1(10), K(11), C(12), c h(13), U(14), b l(15)$, $c t s(16), b t s(17), m l n(18), n b(19), o h(20), r b(21), m w(22), N d(23), s p, Y m$ and $s o$. Each number in parentheses indicates the linkage group to which the marker gene belongs. When a marker gene was recessive to its normal allele, segregation in the back cross of the type $F_{1}$ female $\times$ marker male was tested. When marker genes were dominant one, segregation in the backcross of the type $F_{1}$ female $\times$ normal male was tested.

Determination of sepialumazine. Three $\mathrm{F}_{2}$ hybrid phenotype larvae descended from the cross between Kiuki and normal type silkworms. On the last day of the fourth instar, larvae of each phenotype were dissected and the integument was separated from other tissues and utilized as starting materials. All subsequent procedures were carried out in a dark room: Integument weighing $2 \mathrm{~g}$, was homogenized in five volume of $50 \%$ aqueous ethanol for $4 \mathrm{~min}$. The homogenate was placed in a reflux condenser and heated by a boiling water bath for $20 \mathrm{~min}$. After cooling, the homogenate was centrifuged at $8,000 \mathrm{~g}$ for $20 \mathrm{~min}$. at $4^{\circ} \mathrm{C}$. The residue was again extracted by the same process. The combined supernatant fluid was concentrated to a small volume by a rotary evaporator. About $0.1 \mathrm{~g}$ of ECTEOLA-cellulose powder ( $\mathrm{OH}$ form) was added to the concentrate and the mixture was applied to the top of an ECTEOLA-cellulose column $(\mathrm{pH} 7.0,1 \times 20 \mathrm{~cm})$. Fluorescent bands were detected by an UV light $(365 \mathrm{~nm})$. When the column was washed with water, yellow fluorescent riboflavin and other minor fluorescening compounds were eluted. By successive elution of the column with water, isoxanthopterin was eluted. The 
remaining major column yellow band was eluted with $0.01 \mathrm{~N}$ acetic acid and concentrated to a small volume. The fluid was then placed on a phosphorylated Sephadex G-25 column (fine, $1 \times 20 \mathrm{~cm}$ ). The yellow pigment was eluted from the column with water. After the eluate was dried, it was dissolved in $4 \mathrm{ml}$ of $0.01 \mathrm{M}$ potassium phosphate buffer $\mathrm{pH}$ 7.0. The eluate was identified as sepialumazine and its quantity was estimated by its absorbance at $418 \mathrm{~nm}$ at pH 7.0 (Mazda et al. 1980).

Assay of sepiapterin deaminase activity. On the last day of the fourth instar, larvae were dissected and fat bodies and muscles were carefully removed from the integument with glass spatula. Larval integument weighing $4 \mathrm{~g}$ was homogenized with eight volumes of $0.05 \mathrm{M}$ potassium phosphate buffer, $\mathrm{pH}$ 6.5. The crude extract was partially purified according to the previously described method (Tsusué 1971). The enzyme fraction from the ammonium sulfate step was utilized.

Assay of sepiapterin reductase activity. On the fourth day of the fifth instar, $2 \mathrm{~g}$ of the fat bodies of $\mathrm{F}_{2}$ hybrid silkworms were washed with Ringer's salt solution and homogenized with three volumes of $0.01 \mathrm{M}$ potassium phosphate buffer, $\mathrm{pH}$ 6.5. After centrifugation at $30,000 \mathrm{~g}$ for $90 \mathrm{~min}$., the enzyme activity in the supernatant was assayed by the method described by Katoh (1971).

Protein determination. Protein was determined by the method of Lowry et al. (1959) with bovine serum albumin used as a standard.

\section{RESULTS}

All of the $F_{1}$ larvae obtained from reciprocal crosses between $\mathrm{C} 108$ and Kiuki mutant manifested pale yellow colour. As shown in Table 1, the segregation ratio of yellow and normal phenotypes was approximately 3:1 in C108 $\times$ Kiuki $\mathrm{F}_{2}$ progenies and 1:1 in $\mathrm{C} 108 \times \mathrm{Kiuki} \mathrm{BF}_{1}$, respectively. That a small number of individual larvae of Kiuki phenotype were found in monohybrid experiments does not mean a low viability for the Kiuki larvae. The lower number reflects the difficulty in distinguishing the Kiuki phenotype found in monohybrid larvae from normal phenotype.

Studies were next made on the relationship between the phenotypic segregation of yellow larval colour in the $\mathrm{F}_{2}$ hybrid and integument sepialumazine content. The Bombyx mori strains $\mathrm{C} 108$ and $\mathrm{N} 122$ were used for these studies. As seen in Table 2, the yellow larvae found in $F_{2}$ progeny contained more sepialumazine than did the normal phenotypes. Although the absolute amount of sepialumazine was different when either the Japanese (N122) or Chinese (C108) race was mated with the Kiuki mutant, the ratio of the amounts of the pigment in the three genotype was approximately 5:3:1 in both cases. Silkworm larvae of the Chinese race were rather weakly coloured when 
Table 1. Phenotypic segregation in hybrid of Kiuki and normal silkworm

\begin{tabular}{ccccccc}
\hline \multirow{2}{*}{ Mating } & \multirow{2}{*}{ Batch No. } & \multicolumn{3}{c}{ Phenotype } & \\
\cline { 5 - 6 } & & Yellow & Pale yellow & Normal & $\chi^{2}$ \\
\hline (C108 $\times$ Kiuki) $\mathrm{F}_{2}$ & 1 & 74 & 157 & 79 & 0.213 \\
& 2 & 80 & 163 & 84 & 0.101 \\
$($ C108 $\times$ Kiuki) $\times$ C108 & 1 & 0 & 95 & 120 & 2.91 \\
& 2 & 0 & 123 & 140 & 1.10 \\
\hline
\end{tabular}

Table 2. Sepialumazine content and activities of sepiapterin deaminase and sepiapterin reductase of $F_{2}$ hybrid silkworm

\begin{tabular}{clccc}
\hline Mating & $\begin{array}{c}\text { Phenotype } \\
\text { of } \\
\text { segregated } \\
\text { larva }\end{array}$ & $\begin{array}{c}\text { Sepialumazine } \\
\text { (mg/g integument) }\end{array}$ & $\begin{array}{c}\text { Sepiapterin } \\
\text { deaminase } \\
\text { (units/mg protein) }\end{array}$ & $\begin{array}{c}\text { Sepiapterin } \\
\text { reductase } \\
\text { (units/mg protein) }\end{array}$ \\
\hline (C108 $\times$ Kiuki) $)$ & $\mathrm{F}_{2}$ & & & \\
& Yellow & 0.0355 & 1.05 & 2.03 \\
& Pale yellow & 0.0234 & 0.859 & 1.93 \\
& Normal & 0.00627 & 0.627 & 2.03 \\
(N122 $\times$ Kiuki) & F & & & \\
& Yellow & 0.0665 & 1.26 & 2.23 \\
& Pale yellow & 0.0332 & 1.14 & 2.41 \\
& Normal & 0.0113 & 0.971 & 2.08 \\
\hline
\end{tabular}

compared with Japanese and European races and progenies from a cross with the Japanese race showed a higher sepialumazine content than those from the Chinese race (C108).

For each phenotype of $\mathrm{F}_{2}$ silkworm hybrid, both sepiapterin deaminase and sepiapterin reductase were assayed (Table 2). The results showed that the greater the level of integument sepiapterin deaminase activity, the higher the amount of sepialumazine. Each strain (C108 and N122 in Table 2) had its inherent gene(s) controlling sepiapterin deaminase activity. Kiuki gene increased the enzyme activity over the level which is peculiar to each strain. No correlation, however, was found between integument pigment accumulation and larval fat body sepiapterin reductase activity. In the previous report (Mazda et al. 1980) we demonstrated that yellow larval colour characteristic of the Kiuki mutant is due to the accumulation of sepialumazine in the integument. From Table 1 and Table 2, it is concluded that the yellow larval colour of the Kiuki mutant is controlled by an incomplete dominant gene and that yellow larval colour of this mutant is related to sepiapterin deaminase activity. We will designate the Kiuki mutant by the symbol Sel.

\section{Linkage relation of Sel gene}

Genetic analysis of the linkage relation of the Sel gene was carried out. 
Table 3. Linkage test of Sel (examined in $B F_{1}$ generation)

\begin{tabular}{|c|c|c|c|c|c|}
\hline \multirow{3}{*}{$\begin{array}{c}\begin{array}{c}\text { Linkage } \\
\text { groups } \\
\text { tested }\end{array} \\
2\end{array}$} & \multirow{2}{*}{ Parental genotype } & \multicolumn{4}{|c|}{ Segregation of characters } \\
\hline & & \multicolumn{2}{|c|}{ Sel } & \multicolumn{2}{|c|}{+} \\
\hline & $(+/ Y, \operatorname{Sel} /+) \times(+/+,+/+)$ & $Y \quad 48$ & +47 & $Y \quad 43$ & +51 \\
\hline 3 & $(Z e j+,+/ S e l) \times(+/+,+/+)$ & $Z e 126$ & +137 & Ze 135 & +155 \\
\hline 4 & $(L /+,+/ S e l) \times(+/+,+/+)$ & $L 129$ & +134 & $L 138$ & +134 \\
\hline 5 & $(o c /+,+\mid S e l) \times(o c / o c,+\mid+)$ & +136 & oc 127 & +161 & oc 129 \\
\hline 6 & $\left(E^{E l} /+,+/ S e l\right) \times(+/+,+/+)$ & $E^{E l} 131$ & +132 & $E^{E l} 147$ & +143 \\
\hline 7 & $(q /+,+\mid S e l) \times(q / q,+/+)$ & +113 & $q 93$ & +118 & $q 100$ \\
\hline 8 & $(s t /+,+/ \mathrm{Sel}) \times(s t / s t,+/+)$ & +48 & st $\quad 47$ & +51 & st 43 \\
\hline 9 & $(I-a /+,+\mid S e l) \times(+/+,+/+)$ & $I-a 124$ & +136 & $I \cdot a 127$ & +139 \\
\hline 10 & $(+/ w-1,+/ S e l) \times(w-1 / w-1,+/+)$ & +43 & $w-1 \quad 51$ & +52 & $w-1 \quad 43$ \\
\hline 11 & $(K /+,+\mid \operatorname{Sel}) \times(+/+,+/+)$ & K 127 & +133 & K 153 & +113 \\
\hline 12 & $(+/ C,+/ \mathrm{Sel}) \times(+/+,+/+)$ & $C \quad 28$ & +29 & $C \quad 27$ & +33 \\
\hline 13 & $(c h /+,+/ \mathrm{Sel}) \times(\mathrm{ch} / \mathrm{ch},+/+)$ & +107 & ch 119 & +113 & ch 97 \\
\hline 14 & $(+/ U, S e l /+) \times(+1+,+1+)$ & $U 148$ & +146 & $U 132$ & +124 \\
\hline 15 & $(b l /+, S e l /+) \times(b l / b l,+/+)$ & +128 & $b l \quad 88$ & +132 & $b l 120$ \\
\hline 16 & $(e t s /+, \operatorname{Sel} /+) \times(c t s / c t s,+\mid+)$ & +115 & cts 101 & +120 & cts 132 \\
\hline 17 & $(b t s /+,+\mid S e l) \times(b t s / b t s,+/+)$ & +52 & bts 43 & +43 & bts 51 \\
\hline 18 & $(+\mid m l n, S e l /+) \times(m \ln / m \ln ,+1+)$ & +61 & $m l n \quad 49$ & +62 & $m \ln 77$ \\
\hline 19 & $(+/ n b, S e l /+) \times(n b / n b,+/+)$ & +107 & $n b \quad 70$ & +96 & $n b \quad 62$ \\
\hline 20 & $(+/ o h, S e l)+) \times(o h / o h,+1+)$ & +80 & oh 50 & +101 & oh 58 \\
\hline 21 & $(+/ r b, S e l l+) \times(r b / r b,+/+)$ & +123 & $r b 121$ & +131 & $r b \quad 114$ \\
\hline 22 & $(m w /+,+/ S e l) \times(m w / m w,+/+)$ & +93 & $m w \quad 43$ & +93 & $m w \quad 33$ \\
\hline 23 & $(N d|+,+| S e l) \times(+/+,+/+)$ & $N d \quad 83$ & +58 & $N d \quad 69$ & +121 \\
\hline$?$ & $(+/ s p, S e l /+) \times(s p / s p,+/+)$ & +.44 & $s p \quad 34$ & +25 & $8 p \quad 27$ \\
\hline$?$ & $(+/ Y m,+/ S e l) \times(+/+,+/+)$ & $Y m \quad 40$ & +53 & $Y m \quad 62$ & \\
\hline$?$ & $(s o /+,+/ \mathrm{Sel}) \times(\mathrm{so} / \mathrm{so},+/+)$ & +83 & so 50 & +83 & $80 \quad 36$ \\
\hline
\end{tabular}

Mutant stocks for autosomal marker genes were crossed with Sel, then the segregation in $\mathrm{BF}_{1}$ progenies was examined. An independent relationship between the gene $\mathrm{Sel}$ and chromosome-2 through chromosome-23 was revealed as shown in Table 3. Sel is also independently related to the $s p, Y m$ and $s o$ mutations.

\section{DISCUSSION}

As is well known, sex-chromosome constitution of the silkworm is XY in female and $\mathrm{XX}$ in male, respectively. That the $\mathrm{F}_{1}$ hybrid between $\mathrm{Sel}$ female and $\mathrm{C} 108$ male manifested yellow colour phenotype indicates that the Sel gene is an autosomal and dominant mutation. This was confirmed through 1:1 sex ratio in each phenotypic class segregated in $\mathrm{BF}_{1}$ progenies shown in Tables 1 and 3, though the data were not shown. Linkage test presented in Table 3 clearly showed that the Sel gene is independent of chromosome-2 to chromo- 


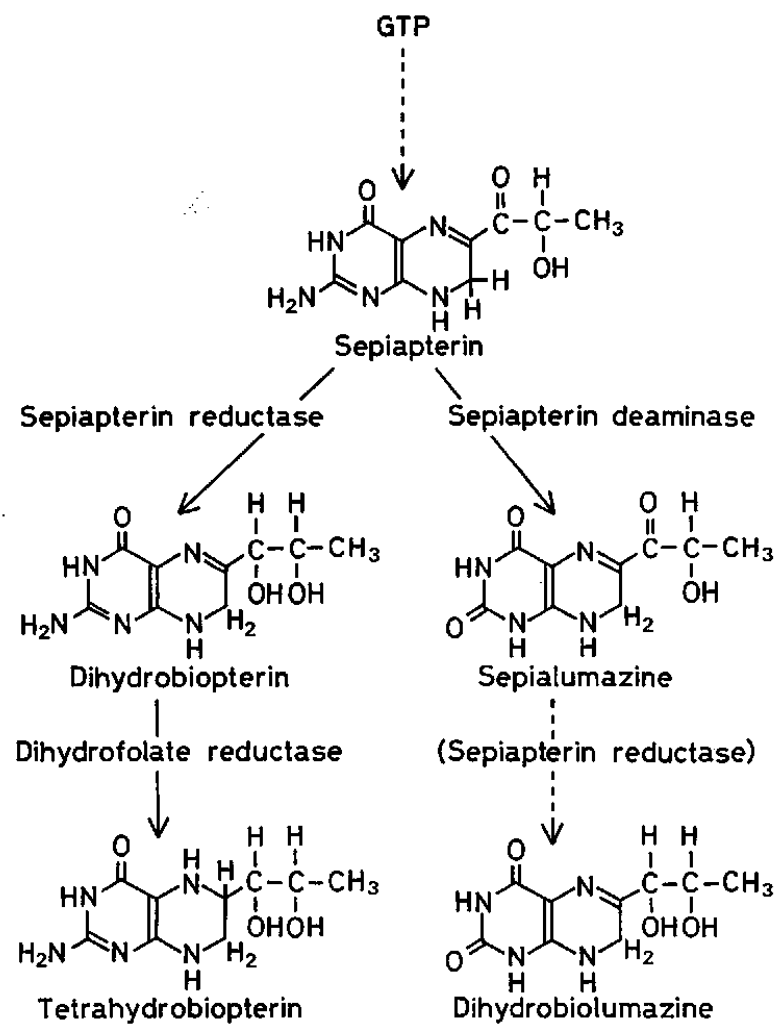

Fig. 1.

some-23 throughout and of the genes for $s p, Y m$ and so. Although twentythree linkage groups have already been established in the silkworm, those three genes belong to some still unknown groups among the remaining five chromosomes. The observed segregation in some crosses were far from the 1:1:1:1 ratio which would have been given when the Sel was not linked to the marker genes. It must be taken into consideration, however, that in Bombyx mori crossing over occurs only on the male side. If $\mathrm{Sel}$ was linked to the marker gene, it would have given the segregation of only parental genetic constitutions.

In the previous paper (Mazda et al. 1980) it was reported that sepialumazine was detected in normal Bombyx mori. The amount of the pigment accumulated in the larval epidermis differs from one strain to another. As seen in Fig. 1, many factors may cause the accumulation of the sepialumazine in larval integuments. First, biosynthesis of sepiapterin in silkworms may be controlled by many genes (Yoshida et al. 1979). Second, the activity of sepiapterin reductase may also affect the quantity of the sepiapterin in integument (Matsubara et al. 1963). Finally, although it is not known whether sepialumazine is further metabolized or not, a decrease of the pigment by 
further metabolism or excretion of the pigment through the malpighian tubules (Tsusué 1971) may also be a controlling force. Since many factors may be involved in the accumulation of the pigment, it is natural that the levels of larval integument sepialumazine are not simply proportional to the activity of sepiapterin deaminase. However, the amount of sepialumazine changed during the larval developmental stages in parallel with deaminase activities provided that the same strain was used (Mazda et al. unpublished). Hence there can be no doubt that the activity of sepiapterin deaminase is an important factor in the accumulation of the pigment in the larval integument. As for relationship between $\mathrm{Sel}$ gene and sepiapterin deminase, at present we have no evidence whether the gene is structural one.

The biochemical significance of sepiapterin deaminase is not yet known. But since sepiapterin is a precursor of tetrahydrobiopterin (Fig. 1) which is a cofactor in the enzymatic hydroxylation of aromatic amino acids (Kaufman 1963), the deaminase may have a role in regulating the levels of this pteridine cofactor. C108 lem (larva homozygous for lem and isogenic to C108) is well viable as compared with $\mathrm{C108}$ strain, in spite of lacking sepiapterin reductase. This fact may indicate the existence of other type pteridine cofactor than tetrahydrobiopterin. Although mechanism of lemon lethal gene have scarcely been known (Sakaguchi and Tsujita 1959), studies on this mechanism will clarify the problem. It was clarified that $\mathrm{Sel}$ gene enhanced sepiapterin deaminase additively over the levels of genetical back ground, but the biological meanings of this gene is not yet known.

In the course of another mating experiment, we found a short winged mutation as a spontaneous occurrence. This short winged mutation was recessive to its normal allele and the gene was tentatively referred to as $s w$. Independent relation of $s w$ to chromosome-1 to chromosome-23 was revealed through successive linkage analyses, though the expressivity of the mutant $s w$ was low. Backeross of the type $(\mathrm{Sel} \times s w)$ 우 $\times s w$ 令 yielded progenies of $104 \mathrm{Sel}+$, $11++$ and $68+s w$ (Doira et al. unpublished). Absence of $s w$ phenotype in $\mathrm{Sel}$ progenies indicates that these two genes are linked with each other. Appearance of ++ phenotype can be explained when we consider low expressivity of $s w$. Thus, the $S e l$ gene represents together with $s w$ one of the hitherto been unknown chromosome to constitute the twenty-fourth linkage group. Further selection for the improvement of low expressivity of $s w$ is necessary, and this linkage phenomenon will be treated elsewhere.

The authors wish to express their gratitude to Dr. W. L. Gyure of the Jersey City Medical Center for his help in preparing this manuscript.

\section{REFERENCES}

Goto, M., Konishi, M., Sugiura, K. and Tsusut, M. (1966) The structure of a yellow pigment from the mutant lemon of Bombyx mori. Bull. Chem. Soc. Japan 39, 929-932.

KaтоH, S. (1971) Sepiapterin reductase from horse liver: Purification and properties of the enzyme. 
Arch. Biochem. Biophys. 146, 202-214.

KaUfMan, S. (1963) The structure of the phenylalanine-hydroxylation cofactor. Proc. Natl. Acad. Sci. USA 50, 1085-1092.

Lowry, O. H., Rosebrough, N. J., FARR, A. L. and Randall, R. J. (1951) Protein measurement with the folin phenol reagent. J. Biol. Chem. 193, 265-275.

Matsubara, M., Tsusut, M. and Akino, M. (1963) Occurrence of two different enzymes in the silkworm, Bombyx mori, to reduce folate and sepiapterin. Nature 199, 908-909.

Mazda, T., Tsusú́, M. and SaKaTe, S. (1978) Characterization of a yellow pigment from a mutant Kiuki of silkworm, Bombyx mori. J. Sericult. Sci. Japan 47, 451-452.

Mazda, T., Tsusué, M. and Sakate, S. (1980) Purification and identification of a yellow pteridine characteristic of the larval colour of the Kiuki mutant of the silkworm, Bombyx mori. Insect Biochem. 10, 357-362.

OGURA, S. (1922) Linkage phenomena between the zebra-factor and others in the silkworm. Jpn. J. Genet. 1, 195-217 (in Japanese).

SAKAGUCHI, B. and Tsusita, M. (1959) Role of eytoplasm in the manifestation of gene-On the lethal yellow gene in silkworm. In Recent Advances in Experimental Morphology (ed. K. TAKeWAKI, M. HARIZUKA and M. FuKaYA), pp. 272-282. Yokendo LTD., Tokyo (in Japanese).

Tsujita, M. (1961) Maternal effect of $+^{\text {lem }}$ gene on pterin reductase of Bombyx mori. Jpn. J. Genet. 36, 337-346.

TsujiTA, M. and SAKAGUCHI, B. (1955) Genetical and biochemical studies of yellow lethal larvae in the silkworm (1). On the nature of pterin obtained from the yellow lethal strain. Jpn.J. Genet. 30, 83-88.

Tsusut, M. (1967) Occurrence of sepiapterin deaminase in the silkworm, Bombyx mori. Experientia 23, 116-117.

TsuSUÉ, M. (1971) Studies on sepiapterin deaminase from the silkworm, Bombyx mori. $J$. Biochem. 69, 781-788.

TsusuÉ, M. and AKINo, M. (1965) Yellow pterin in mutant lemon of silkworm and mutant sepia of D. melanogaster. Zool. Mag. 74, 91-94 (in Japanese with English summary) .

Tsusut, M. and MAzDA, T. (1967) Occurrence of sepiapterin deaminase in the normal type silkworm, Bombyx mori. Experientia 33, 854-855.

Yoshida, T., Masada, M., Tomino, S. and Akino, M. (1979) Biosynthesis of sepiapterin. Zool. Mag. 88, 559 (in Japanese). 